JAMA Neurol. 2015 July ; 72(7): 743-744. doi:10.1001/jamaneurol.2014.4275.

\title{
ALS: one size does not fit all
}

\author{
Stephen A. Goutman, $\mathbf{M D}^{1}$ and Eva L. Feldman, MD, $\mathbf{P h D}^{1}$ \\ ${ }^{1}$ Department of Neurology, University of Michigan, Ann Arbor, MI, 48109
}

The noted French neurologist, Jean- Marie Charcot (1825-1893), is acclaimed for his studies on the motor system and unifying previously disparate entities into one disorder, amyotrophic lateral sclerosis (ALS). His careful work correlating neurological signs with anatomy lead to the concept that a spectrum of neurological disorders including progressive muscular atrophy, progressive bulbar palsy and primary lateral sclerosis represented a more singular entity, ALS. Charcot summarized his ideas in a highly cited review in 1874 and this idea has dominated medical nosology for 140 years.(1)

Today in clinical practice, neurologists easily recognize the various "subtypes" of ALS which, in addition to the above, also include the flail arm and leg variants(2). So, we now question: is ALS one disorder as Charcot would contend (3) or has the clock turned back? Is the essential question now: are these subtypes of one disease or multiple different diseases with one final common anatomical and clinical endpoint?

The rapid advancement in genetic discoveries clearly points to multiple different disorders, with ALS being much more a syndrome than a singular distinct entity. For example, the C9orf72 hexanucleotide expansion, the genetic mutation currently most commonly associated with familial and sporadic ALS, represents a clinical spectrum with the extremes representing ALS or frontotemporal dementia and the middle region representing patients having classical symptoms of ALS combined with executive dysfunction and a reduced survival. On the other hand, phenotypes within SOD1, the first ALS gene identified, range from a short and rapid course to a prolonged clinical course depending on the specific mutation. Finally, patients with fused in sarcoma (FUS) affect younger persons and have a more rapid progression(2).

We contend that the persistence in considering ALS a singular disorder is a major reason that many of the previous therapeutic drug trials have failed. These drugs may benefit some ALS subtypes but have no effect on others. Furthermore, the reliance on the SOD1 mouse model for drug development may not accurately assess all ALS disease mechanisms and it is possible that effective drugs may not even be tested in patients if they do not show efficacy in these mouse models. A more rational approach lies in defining ALS subtypes based on both clinical presentation and when applicable genetic variability. With the understanding that ALS is not a singular disorder, the ALS research community needs to move away from

Corresponding Author: Stephen A. Goutman, MD, Department of Neurology, University of Michigan, F2647 UH South, SPC 5223, 1500 E. Medical Center Drive, Ann Arbor, MI 48109-5223, Phone: 734-936-8586, Fax: 734-936-5185, sgoutman@med.umich.edu. Author's Contributions: Both authors contributed equally to this paper.

Conflict of Interest Disclosures: The authors have no disclosures to report. 
the idea that there is one ideal clinical trial endpoint. We agree that the application of new efficacy outcome measures combined with pharmacodynamic markers will improve the ability to detect meaningful clinical outcomes and that these endpoints may need to be stratified by disease heterogeneity (4). Similar views have recently been provided by the Food and Drug Administration (FDA) (http://www.regulations.gov/\#! documentDetail;D=FDA-2013-N-0035-0272). In their response to a February 2013 hearing on drug treatment for ALS, the FDA agreed that disease heterogeneity may impact clinical trial outcomes and methods that can identify these subgroups for an efficacy analysis would be supported. Furthermore, the FDA indicated that is it open to alternative clinical trial endpoints and novel study designs that apply endpoints other than the more traditional survival and functional scale outcomes.

ALS patients are also recognizing the importance of incorporating the heterogeneity of the disease and wish to work with the ALS research community to utilize these differences in the development of clinical trials. In a personal communication, Josh von Schaumburg, a member of the ALS Emergency Treatment Fund (http://alsetf.org/research/), questioned whether subgroup analyses in prior clinical trials would have identified an effective treatment for a family member with young bulbar-onset disease. Furthermore, considering ALS patients' enthusiasm for participating in clinical trials at all stages of their disease, the use of disease phenotypic variability combined with new biomarkers and genotyping may aid in the collection of phase II outcomes unique from outcomes for phase III studies, so that phase II studies do not simply represent underpowered phase III trials.

We believe that the proposed mechanism underlying the therapeutic intervention must guide the choice of clinical endpoints. We completed a Phase 1(5) and Phase 2 trial of intraspinal stem cell transplantation in ALS. Our final cohort of 3 subjects in the Phase 2 trial underwent both lumbar and cervical transplantation and received a total of 16 million neural stem cells. The therapeutic rationale underlying transplantation is the preservation of motor neurons in and around the transplanted regions. Our proposed therapy would not benefit bulbar patients as the stem cells cannot be delivered to the most affected regions nor would they benefit those with more advanced disease stages as the stem cells do not replace lost motor neurons. Moreover, from a clinical trial design standpoint this same group of patients is at a stage in their disease where current outcome measures may not be robust enough to detect a treatment effect. At the same time, we as investigators recognize the desire of patients to participate in these studies regardless of the disease severity. Participation in clinical research brings patients hope and also provides a means for them to feel that they are helping to advance ALS knowledge. As we design the next Phase 2/3 trial, we are encountering the difficulties and conundrums of previous clinical trials that had to grapple with the fact that ALS is not one disease, but a syndrome. While we aim to use novel endpoints as a means to assess efficacy of the stem cells, we may need to rely on more established measures in a group of patients most likely to demonstrate a statistically meaningful disease modification.

If we are to make therapeutic progress, we need to reassess our position as a neurological community and come to grips with the idea that "one size does not fit all" when we approach clinical trial designs in ALS. This will not be without its own challenges, 
especially in clinical trial recruitment and powering of studies. Yet without a rational more strategic approach, future ALS clinical trials will fail as have their predecessors. The nagging question remains: have previous therapies failed because of our lack of defining disease subtypes and the selection of the wrong clinical endpoints? More importantly, as we shift from the medical nosology of Charcot of 140 years ago to a nosology based on a combination of rapidly advancing genetics and imaging, can our increased understanding of ALS as a syndrome lead to improved trial designs with relevant clinical outcomes? As a neurological community, we need to readdress our diagnostic and therapeutic approaches because when it comes to ALS, "one size does not fit all."

\section{Acknowledgments}

Funding support was provided by the National Institutes of Health (R01 NS077982, R01 NS08230401A1), the Centers for Disease Control and Prevention/Agency for Toxic Substances and Disease Registry (Contract \# 200-2013-56856), and the A. Alfred Taubman Medical Research Institute. The funders had no role in study design, data collection and analysis, decision to publish, or preparation of the manuscript.

\section{References}

1. Charcot J-M. De la sclérose latérale amyotrophique. Prog Med. 1874:325-7. 41-42, 453-5.

2. Al-Chalabi A, Hardiman O. The epidemiology of ALS: a conspiracy of genes, environment and time. Nat Rev Neurol. 2013 Nov; 9(11):617-28. Epub 2013/10/16. eng. [PubMed: 24126629]

3. Vucic S, Rothstein JD, Kiernan MC. Advances in treating amyotrophic lateral sclerosis: insights from pathophysiological studies. Trends in neurosciences. 2014 Jun 10.

4. Berry JD, Cudkowicz ME, Shefner JM. Predicting success: optimizing phase II ALS trials for the transition to phase III. Amyotroph Lateral Scler Frontotemporal Degener. 2014 Mar; 15(1-2):1-8. Epub 2014/03/05. eng.

5. Feldman EL, Boulis NM, Hur J, Johe K, Rutkove SB, Federici T, et al. Intraspinal neural stem cell transplantation in amyotrophic lateral sclerosis: phase 1 trial outcomes. Ann Neurol. 2014 Mar; 75(3):363-73. [PubMed: 24510776] 